\title{
Die Konzessionsbereitschaft von Bewerbern und Beschäftigten nach den Hartz-Reformen
}

\begin{abstract}
Die Wirkungen der Hartz-Reformen sind ein viel beachtetes Thema in Forschung und Öffentlichkeit. Wenig untersucht sind bisher jedoch die Verhaltensänderungen, die sie bei unterschiedlichen Personengruppen hervorgerufen haben könnten. Dieser Beitrag untersucht Determinanten für Veränderungen im Verhalten arbeitsloser Bewerber und Beschäftigter aus der betrieblichen Perspektive. ${ }^{1}$ Wie hat sich die Konzessionsbereitschaft hinsichtlich ihrer Arbeit und ihrer Entlohnung verändert? Welche Einflussfaktoren für eine gestiegene Konzessionsbereitschaft und auch Hinweise auf Verhaltensänderungen aufgrund der Reformen lassen sich identifizieren. ${ }^{2}$
\end{abstract}

\section{Einleitung}

Zwischen den Jahren 2001 und 2005 befand sich Deutschland in einer schwierigen ökonomischen Lage: Der Arbeitskräftebedarf war rückläufig, die Zahl der Arbeitslosen stieg auf einen sehr hohen Wert und das wirtschaftliche Wachstum war gebremst. Trotz hoher Arbeitslosigkeit gab es aber offene Stellen, die Unternehmen nicht besetzen konnten (Kettner/Spitznagel 2006). Diese Entwicklungen gaben Anlass, die strukturellen Probleme des Arbeitsmarktes anzugehen, was zur Umsetzung der weitreichendsten Arbeitsmarktreformen seit Jahrzehnten führte. Im Rahmen der Agenda 2010 wurden zwischen 2003 und 2005 die sogenannten Hartz-Reformen verabschiedet.

Diese Reformen, insbesondere die 2005 eingeführte Hartz-IV-Reform, setzten auf die Verkürzung der Arbeitslosigkeitsdauer und damit die Verringerung der Folgeeffekte von Langzeitarbeitslosigkeit. Dies sollte unter anderem mit der Einführung von Zielvereinbarungen, verschärften Zumutbarkeits- und Sanktionsregelungen erreicht werden. Wenn die Reformen zu einer Aktivierung Arbeitsloser geführt haben, müsste dies in Verhaltensänderungen dieser Personen deutlich werden. Es müsste ein verstärktes Bemühen um eine Beschäftigung und eine stärkere Bereitschaft, Arbeitsplätze zu akzeptieren, die nicht den ursprünglichen Vorstellungen entsprechen, sichtbar werden. Auch ist davon auszugehen, dass die Anspruchslöhne sinken, da das Arbeitslosengeld ab 2005 auf (in der Regel) ein Jahr begrenzt wurde. Danach erhalten Arbeitslose eine Grundsicherung (Arbeitslosengeld
II), die etwa der früheren Sozialhilfe entspricht und nicht von der Höhe des vorherigen Erwerbseinkommens abhängt. Im Falle von Vermögen oder ausreichendem Partnereinkommen besteht kein Anspruch auf Arbeitslosengeld II (Zweites Buch Sozialgesetzbuch). Mit der stärkeren Aktivierung von Arbeitslosen war auch die Hoffnung verbunden, dass es Unternehmen gelingt, bis dato nicht besetzbare bzw. schwer besetzbare Stellen leichter besetzen zu können.

Von sinkenden Anspruchslöhnen Arbeitsloser könnten auch Beschäftigte indirekt betroffen sein. Wenn arbeitslose Bewerber konzessionsbereiter sind und beispielsweise einen geringeren Lohn akzeptieren und zu diesem eingestellt werden, dann stehen sie in Konkurrenz zu den bereits Beschäftigten. Diese dürften jedoch auch direkt durch die Reformen beeinflusst worden sein. Durch die Begrenzung des Arbeitslosengeldes auf ein Jahr hat sich das Alternativeinkommen im Falle länger andauernder Arbeitslosigkeit deutlich verringert. Das finanzielle Risiko eines Arbeitsplatzverlustes könnte insbesondere im konjunkturschwachen Jahr 2005 auch bei Beschäftigten zu zurückhaltenden Lohnforderungen und stärkerer Leistungsbereitschaft geführt haben.

Ein empirischer Nachweis von Verhaltensänderungen ist für beide Personengruppen nur schwer zu erbringen. In der Regel bedarf es dafür längerer Zeitreihen, um diese auf einen Strukturbruch zu testen. Kurzfristig können Befragungsdaten jedoch Anhaltspunkte liefern. Deshalb wird in diesem Beitrag auf Daten der Jahre 2005 und 2006 der IAB-Erhebung des gesamtwirtschaftlichen Stellenangebots zurückgegriffen.

Im Folgenden wird erstens untersucht, ob Betriebe in diesen Jahren eine gestie- gene Konzessionsbereitschaft arbeitsloser Bewerber beobachtet haben. Dabei wird angenommen, dass die Reformen das Verhalten der Bewerber in der Wahrnehmung der Betriebe verändert haben. Zweitens wird analysiert, ob Verhaltensänderungen in der Belegschaft beobachtet wurden. Auch hier wird angenommen, dass die institutionellen Änderungen einen Einfluss ausgeübt haben, der zu erhöhter Konzessionsbereitschaft geführt hat.

Mit der Einführung der Hartz-IVReform im Januar 2005 war der Reformprozess (vorerst) abgeschlossen. Dieser letzte Reformbaustein brachte auch die Änderungen mit sich, die vornehmlich zu einer höheren Konzessionsbereitschaft beitragen können: die Zusammenlegung der Arbeitslosenhilfe mit der Sozialhilfe

\footnotetext{
1 Aus Gründen der Lesbarkeit verzichtet der Tex bei den Gruppenbezeichnungen auf die weibliche Form. Wo möglich werden geschlechtsneutrale Begriffe verwendet; generell sind Bewerber und Beschäftigte unabhängig vom Geschlecht gemeint.

2 Die Autorinnen danken Dr. Sabine Klinger, Michael Stops und zwei anonymen Gutachtern in den WSI-Mitteilungen für sehr hilfreiche Hinweise.
}

Martina Rebien, Soziologin,
wissenschaftliche Mitarbeiterin am Institut
für Arbeitsmarkt- und Berufsforschung
(IAB) in Nürnberg. Arbeitsschwerpunkte:
Netzwerke bei Stellenbesetzungsprozessen,
betriebliche Auswirkungen der Hartz-
Reformen.
e-mail: martina.rebien@iab.de
Anja Kettner, Dipl.-Volkswirtin, leitet am
IAB die Erhebung des gesamtwirtschaftlichen
Stellenangebots. Arbeitsschwerpunkte:
Determinanten der Arbeitskräftenachfrage,
Stellenbesetzungsprozesse.
e-mail: anja.kettner@iab.de


und die Verkürzung der Bezugsdauer des Arbeitslosengeldes. Die Reform wurde in der Öffentlichkeit viel diskutiert und es kann angenommen werden, dass Betriebe aufgrund der intensiven Debatte für eine Zunahme von Konzessionsbereitschaft sensibilisiert waren.

Im zweiten Abschnitt wird ein Überblick über die empirische und die theoretische Literatur gegeben. Nach einer Beschreibung der Daten (Abschnitt 3) werden bereits vorhandene deskriptive Ergebnisse aus der Erhebung zusammengefasst (Abschnitt 4). In Abschnitt 5 wird auf die Spezifikation der verwendeten logistischen Regressionsmodelle eingegangen, deren Ergebnisse im Anschluss präsentiert (Abschnitt 6) und in Abschnitt 7 abschließend zusammengefasst werden.

\section{Zur Konzessionsbereit- schaft: Theorie und Empirie}

\subsection{KONZESSIONSBEREITSCHAFT VON ARBEITSLOSEN}

Laut der ökonomischen Suchtheorie werden Arbeitsuchende dann einen Job akzeptieren, wenn das Angebot mindestens dem Reservationslohn des Suchenden entspricht. Dabei wird regelmäßig angenommen, dass die Schwelle dieses fiktiven Reservationslohns mindestens auf gleicher Höhe mit dem aktuellen Einkommen liegt und sowohl von den Persönlichkeitsmerkmalen als auch der Nachfrage nach Arbeit abhängig ist. Im Falle arbeitsloser Arbeitsuchender würde das aktuelle Einkommen den Transferleistungen entsprechen. Ist der Reservationslohn relativ hoch, so wird angenommen, dass eine Person weniger intensiv nach Arbeit sucht, sofern sie davon ausgehen muss, dass er auf dem Arbeitsmarkt nicht realisiert werden kann (vgl. Christensen 2003). Im Zuge der Arbeitsmarktreform Hartz IV wurde das Arbeitslosengeld auf einen Bezugszeitraum von (in der Regel) einem Jahr gekürzt. Im Anschluss erhalten Arbeitslose eine Grundsicherung, welche in den meisten Fällen einen deutlichen Einkommenseinbruch bedeutet. Theoretisch kann nun angenommen werden, dass diese geringeren Transferzahlungen und die verkürzte Bezugsdauer des Arbeitslosengeldes zu einem sinkenden Reservationslohn führen. Arbeitslose müssten dadurch veranlasst werden, intensiver nach einem Job zu suchen und in stärkerem Maße Einschränkungen zu akzeptieren.

Bereits in den 1980er und 1990er Jahren wurden Änderungen der Bezugsdauer des Arbeitslosengeldes in Deutschland beschlossen. Zwischen 1985 und 1987 wurden die Bezugsdauern für Ältere (ab 43 Jahre) sukzessive verlängert, während sie für jüngere Arbeitslose unverändert blieben. Ab einem Alter von 55 Jahren konnte das Arbeitslosengeld bis zu 32 Monate lang bezogen werden. In den 1990er Jahren wurde diese Regelung teilweise rückgängig gemacht. $\mathrm{Ab}$ 1997 wurde nur noch den über 57-Jährigen eine Bezugsdauer dieser Länge gewährt.

Die Auswirkungen dieser Reformen auf die Eintrittswahrscheinlichkeit in den Arbeitsmarkt wurden mit widersprüchlichen Ergebnissen untersucht. So findet Hunt (1995), dass die Verlängerung der Bezugsdauer des Arbeitslosengeldes stark verlängernd auf die Dauer der Arbeitslosigkeit wirkt. Decressin (2001) untersucht die gleichen Veränderungen. Mit dem Unterschied, dass er die Gruppe der untersuchten Personen auf jene beschränkt, die tatsächlich berechtigt sind, Arbeitslosengeld zu erhalten, kommt er zu dem Ergebnis, dass es zwischen den institutionellen Änderungen und der Arbeitslosigkeitsdauer keinen systematischen $\mathrm{Zu}$ sammenhang gibt (für einen umfassenden Überblick auch über weitere Studien siehe Fitzenberger/Wilke 2009; vgl. hierzu auch Fehr/Vobruba in diesem Heft). Eindeutige Hinweise, dass diese Reformen einen negativen Einfluss auf die Suchintensität bei (älteren) Arbeitslosen hatten, konnten nicht gefunden werden.

Bei den Arbeitsmarktreformen, die zwischen 2003 und 2005 auf dem deutschen Arbeitsmarkt eingeführt wurden, gingen die institutionellen Änderungen jedoch über die verkürzte Bezugsdauer des Arbeitslosengeldes hinaus. Jacobi und Kluve (2007) identifizieren drei Kernelemente der Hartz-Reformen, die Einfluss auf die Beschäftigungswahrscheinlichkeit von Kurz- und Langzeitarbeitslosen haben könnten. Das erste Element bildet dabei die höhere Effektivität und Effizienz der Arbeitsvermittlung, die beispielsweise durch eine Kundensegmentierung in der Arbeitsvermittlung erreicht werden. Das zweite Kernelement steht in engen Zusammenhang mit dem Prinzip des „Förderns und Forderns". So sollte durch die Ausweitung aktiver Arbeitsmarktpolitik, wie die Förderung der Selbstständigkeit, aber auch verschärfte Zumutbarkeitsregelungen und die Zusammenlegung von Arbeitslosenund Sozialhilfe, die Eigeninitiative und Eigenverantwortung Arbeitsloser gestärkt werden. Ein drittes Kernelement finden Jacobi/Kluve (ebd.) in der Lockerung restriktiver Arbeitsmarktregulierungen wie den Regelungen befristeter Beschäftigung oder dem Kündigungsschutz. Das zweite Kernelement ist für die folgenden Analysen von besonderer Bedeutung. Im Zuge der Reformen wurden sukzessive die Anforderungen an Arbeitslose verschärft, aber auch Fördermöglichkeiten ausgebaut (vgl. für einen detaillierten Überblick Klinger/ Rothe 2010).

$\mathrm{Ob}$ und inwiefern Arbeitsmarktreformen einen Einfluss auf das Verhalten von Arbeitslosen haben, wurde vor allem auch für Dänemark untersucht. Dort wurde Mitte der 1990er Jahre das sogenannte Flexicurity-Modell umgesetzt, das ein Vorbild für die deutsche Agenda 2010 war. Es beinhaltet im Wesentlichen einen Interessenausgleich zwischen Arbeitgebern und Arbeitnehmern. Während Arbeitgebern dabei eine Lockerung des Kündigungsschutzes zugute kommt, sind Arbeitnehmer durch eine zwar kurzfristige, aber hohe materielle Unterstützung im Falle von Arbeitslosigkeit und die Unterstützung zur Wiedereingliederung in Arbeit abgesichert (vgl. Braun 2001). Dieses Modell enthält Elemente, die mit den HartzReformen in Deutschland vergleichbar sind: beispielsweise Aktivierung anstelle von rein passiver Leistungszahlung oder Elemente, die den Arbeitsförderungsmaßnahmen entsprechen (Andersen/Svarer 2007). Beide Reformen folgen dem Prinzip des „Förderns und Forderns“.

Die Ergebnisse verschiedener Studien für Dänemark legen nahe, dass unter anderem der Einsatz von aktivierenden arbeitsmarktpolitischen Maßnahmen einen positiven Effekt auf die Wiedereingliederung in Arbeit hat (Abbring et al. 2005; Svarer 2007; Geerdsen 2006). Für Deutschland kommen Bender et al. (2008) zu dem Ergebnis, dass sich die Bereitschaft zu Lohnkonzessionen mit der Einführung der Hartz-IV-Reform (noch) nicht wesentlich geändert hat. Da die Studie sich auf die Befragung von Arbeitslosen im Rechtskreis SGB II konzentriert, können mögliche Effekte in einer anderen Gruppe von Arbeitslosen nicht 
beobachtet werden. Fahr und Sunde (2009) zeigen aber, dass die Arbeitsmarktreformen Hartz I bis III den Matchingprozess in Deutschland beschleunigt haben - eine erhöhte Suchintensität der Arbeitslosen könnte die Ursache hierfür sein.

\subsection{KONZESSIONSBEREITSCHAFT VON BESCHÄFTIGTEN}

Explizite Ergebnisse, inwiefern Arbeitsmarktreformen das Verhalten von Beschäftigten beeinflussen, liegen bisher nicht vor. Es kann jedoch angenommen werden, dass eine hohe Konzessionsbereitschaft arbeitsloser Bewerber den Druck auf die Beschäftigten erhöhen kann. Im Rahmen der Insider-Outsider-Theorie wird von einer weitreichenden Marktmacht Beschäftigter (Insider) ausgegangen, die im Wesentlichen auf der Höhe von Entlassungs-, Einstellungs- und Einarbeitungskosten beruht (Lindbeck/Snower 2001). Wenn arbeitslose Bewerber (Outsider) konzessionsbereiter sind und beispielsweise einen geringeren Lohn oder unterwertige Arbeit akzeptieren, stehen sie in Konkurrenz zu den bereits Beschäftigten. Die Position der Beschäftigten würde dann abgeschwächt, wenn sich die Kostenunterschiede zwischen der Entlassung eines Insiders und der Einstellung eines Outsiders bei höherer Konzessionsbereitschaft der Outsider verringern würden. In diesem Fall würden die Beschäftigten einen Teil ihrer Marktmacht verlieren. Dies dürfte insbesondere im Bereich der gering Qualifizierten bzw. bei Tätigkeiten der Fall sein, bei denen die Einarbeitung wenig Kosten verursacht. Da der größte Teil der Arbeitslosen in Deutschland im geringqualifizierten Bereich zu finden ist (Reinberg/Hummel 2007), würde für Beschäftigte in diesem Bereich die Gefahr bestehen, durch neue (zuvor arbeitslose) Mitarbeiter ersetzt zu werden.

Verstärkend könnte hinzukommen, dass die Einführung der Reformen in wirtschaftlich schwachen Jahren erfolgte. Der befürchtete Arbeitsplatzverlust ist dann wahrscheinlicher, und die Chancen auf einen neuen Arbeitsplatz sind geringer. Hinzu kommt, dass der größere Lohnabstand zwischen Arbeitslosengeld II und dem Einkommen aus Beschäftigung Auswirkungen auf das Verhalten der Beschäftigten haben könnte - die verkürzte Bezugsdauer des Arbeitslosengeldes könnte so indirekt Einfluss auf ihr Verhalten haben.
Auf der Grundlage eines theoriegestützten Maßes entwickeln Ashford et al. (1989) zehn Hypothesen zu den Ursachen für Arbeitsplatzunsicherheit. Danach führt eine steigende Zahl organisatorischer Umbrüche zu steigender Arbeitsplatzunsicherheit. Als solche werten Beschäftigte z.B. die Zahl der betrieblichen Entlassungen (Ashford et al. 1989, S.816 nach Greenhalgh/ Rosenblatt 1984), was besonders in Phasen der Rezession von Bedeutung sein dürfte. Gleichzeitig kann ein hohes Niveau regionaler Arbeitslosigkeit zu einer gestiegenen Arbeitsplatzunsicherheit führen (Clark et al. 2009). Auch die Unsicherheit Beschäftigter über ihre individuelle Position im Betrieb ist bedeutsam. Dies dürfte vor allem Personen betreffen, die befristet oder prekär beschäftigt sind, da ihre Integration in den Betrieb geringer ist als die Beschäftigter im „Normalarbeitsverhältnis“. Die Zahl der Geringverdiener in Deutschland ist von 1995 bis 2006 um etwa $43 \%$ gestiegen (Kalina/Weinkopf 2008). Dieser Zuwachs impliziert, dass sich das Feld der prekären Beschäftigungsverhältnisse erweitert und die Lohnungleichheit zugenommen hat (ILO 2008; Bosch et al. 2008).

Ashford et al. (1989) konnten keine Hinweise darauf finden, dass eine wachsende Arbeitsplatzunsicherheit zu einer geringeren Leistungsbereitschaft Beschäftigter führt. Umgekehrt könnte sie jedoch zu gestiegener Leistungsbereitschaft führen, wenn sich die Lage auf dem Arbeitsmarkt verschlechtert. Schramm (1992) identifiziert neben der Angst vor dem Arbeitsplatzverlust eine zweite Komponente von Arbeitsplatzunsicherheit: die individuellen Einschätzungen der Chancen auf dem Arbeitsmarkt. Seine Ergebnisse zeigen den Einfluss dieser Unsicherheit auf das Verhalten Beschäftigter: Sie stehen in größerer Konkurrenz zueinander, lassen sich disziplinieren und verhalten sich den Vorgesetzten gegenüber unterwürfig (Schramm 1992). Ihre Bereitschaft zu Konzessionen ist also höher.

\section{Erhebung des gesamt- wirtschaftlichen Stellen- angebots}

Um Hinweise auf Verhaltensänderungen bei arbeitslosen Bewerbern und Beschäftigten nach Einführung der Reformen zu finden, werden Daten der IAB-Erhebung des gesamtwirtschaftlichen Stellenangebots genutzt. Diese Querschnittsbefragung wird jährlich im vierten Quartal vom IAB durchgeführt.

Dafür wird eine repräsentative Stichprobe von Betrieben aus der Beschäftigtenstatistik der Bundesagentur für Arbeit gezogen. Sie ist disproportional nach 28 Wirtschaftszweigen und acht Betriebsgrößenklassen, getrennt für Ost- und Westdeutschland, geschichtet. Die Stichprobe beinhaltet etwa 75.000 Betriebe und Verwaltungen mit mindestens einem sozialversicherungspflichtig Beschäftigten. Der Rücklauf wird ständig auf seine Repräsentativität kontrolliert und beläuft sich auf etwa $20 \%$ (für detailliertere Informationen siehe Kettner et al. 2011).

Die Erhebung dient unter anderem dazu, Informationen über betriebliche Einschätzungen $\mathrm{zu}$ aktuellen arbeitsmarktpolitischen Instrumenten zu erhalten. Seit 2005 wird in diesem Rahmen die Hartz-IV-Reform thematisiert. Die Befragungswellen der Jahre 2005 und 2006 beinhalten zwei Frageblöcke, in denen Betriebe einschätzen sollen, inwiefern sie bei arbeitslosen Bewerbern und ihrer Belegschaft Verhaltensänderungen jeweils gegenüber dem Vorjahr festgestellt haben.

Im ersten Fragenblock äußerten die Betriebe ihre Einschätzung, ob die Bereitschaft arbeitsloser Bewerber gestiegen, unverändert oder gesunken sei, einen Arbeitsplatz unterhalb der eigentlichen Qualifikation anzunehmen, Zugeständnisse bei der Entlohnung zu machen oder besondere Arbeitsbedingungen zu akzeptieren.

Der zweite Fragenblock betraf die Einschätzung der Betriebe, ob die Bereitschaft ihrer Belegschaft gestiegen, unverändert oder gesunken sei, leistungsbereiter zu sein, längere oder geänderte Arbeitszeiten oder Einschränkungen bei der Entlohnung oder den Zulagen zu akzeptieren.

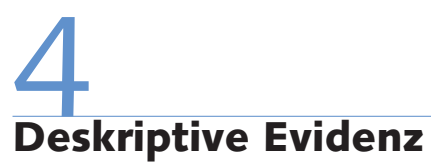

Bereits aus deskriptiven Analysen (Kettner/Rebien 2007) lassen sich Hinweise ableiten, ob sich das Verhalten von Bewerbern und Belegschaften in den Befragungsjahren verändert hat.

Von den Betrieben, die in den vorangegangenen zwölf Monaten vor der Be- 


\section{Tabelle 1: Veränderung der Konzessionsbereitschaft arbeitsloser \\ Bewerber - Anteil an Betrieben mit Neueinstellungen - in \% -}

\begin{tabular}{llcr} 
Die Bereitschaft der Bewerber,... & & $\begin{array}{c}\text { Veränderung 2005 } \\
\text { gegenüber 2004* }\end{array}$ & $\begin{array}{c}\text { Veränderung 2006 } \\
\text { gegenüber 2005* }\end{array}$ \\
\hline eine unterwertige Tätigkeit anzunehmen, & gestiegen & 26 & 20 \\
ist... & gleich geblieben & 62 & 66 \\
& gesunken & 12 & 14 \\
& gesamt & 100 & 100 \\
\hline Zugeständnisse bei der Entlohnung zu & gestiegen & 32 & 23 \\
machen, ist... & gleich geblieben & 55 & 62 \\
& gesunken & 14 & 15 \\
& gesamt & 100 & 100 \\
\hline besondere Arbeitsbedingungen zu & gestiegen & 29 & 20 \\
akzeptieren, ist... & gleich geblieben & 56 & 64 \\
& gesunken & 15 & 16 \\
& gesamt & 100 & 100 \\
\hline
\end{tabular}

* gerundete Werte.

Quelle: IAB-Erhebung des gesamtwirtschaftlichen Stellenangebots 2005 und 2006; Gewichtung: Betriebskonzept.

WSI MITTELUNGEN

Tabelle 2: Veränderung der Konzessionsbereitschaft in der Belegschaft - Anteil an allen Betrieben - in \% -

\begin{tabular}{|c|c|c|c|}
\hline \multicolumn{2}{|c|}{ Die Bereitschaft der Belegschaft... } & \multirow{2}{*}{$\begin{array}{c}\begin{array}{c}\text { Veränderung } 2005 \\
\text { gegenüber } 2004\end{array} \\
33\end{array}$} & \multirow{2}{*}{$\begin{array}{c}\text { Veränderung } 2006 \\
\text { gegenüber } 2005 \\
29\end{array}$} \\
\hline zu höherer Leistung, ist... & gestiegen & & \\
\hline & gleich geblieben & 62 & 66 \\
\hline & gesunken & 5 & 5 \\
\hline & gesamt & 100 & 100 \\
\hline \multirow{4}{*}{$\begin{array}{l}\text { längere oder geänderte Arbeitszeiten zu } \\
\text { akzeptieren, ist... }\end{array}$} & gestiegen & 37 & 30 \\
\hline & gleich geblieben & 57 & 63 \\
\hline & gesunken & 6 & 7 \\
\hline & gesamt & 100 & 100 \\
\hline \multirow{4}{*}{$\begin{array}{l}\text { Einschränkungen bei der Entlohnung zu } \\
\text { akzeptieren, ist... }\end{array}$} & gestiegen & 21 & 14 \\
\hline & gleich geblieben & 68 & 73 \\
\hline & gesunken & 11 & 13 \\
\hline & gesamt & 100 & 100 \\
\hline
\end{tabular}

Quelle: IAB-Erhebung des gesamtwirtschaftlichen Stellenangebots 2005 und 2006; Gewichtung: Betriebskonzept.

WSI MITTELLUNGEN

fragung im Jahr 2005 Neueinstellungen vorgenommen hatten, berichten $26 \%$ über eine gestiegene Bereitschaft arbeitsloser Bewerber, einen Arbeitsplatz unterhalb der erworbenen Qualifikation anzunehmen (Tabelle 1). Ein Jahr später sind es noch $20 \%$. Weiterhin beobachtet etwa jeder dritte Betrieb im Jahr 2005 eine gestiegene Bereitschaft, Zugeständnisse bei der Entlohnung zu machen. Die Bereitschaft, besondere Arbeitsbedingungen zu akzeptieren, wird von $29 \%$ der Betriebe festgestellt. Es zeigt sich deutlich, dass wesentlich seltener eine gesunkene Konzessionsbereitschaft beobachtet wird.

Im Hinblick auf Verhaltensänderungen der Belegschaft (Tabelle 2) berichtet etwa jeder dritte Betrieb in beiden Befragungsjahren von einer gestiegenen Bereitschaft zu höherer Leistung. Mehr als ein Drittel konstatiert im Jahr 2005 eine gestiegene Bereitschaft, längere oder geänderte Arbeitszeiten zu akzeptieren. Im gleichen Jahr geben etwa $21 \%$ der Betriebe an, ihre Belegschaft sei eher als im Vorjahr bereit, Einschränkungen bei der Entlohnung zu akzeptieren.

Es wird deutlich, dass der größte Teil der Betriebe keine Verhaltensänderungen in den Gruppen feststellt. Bis zu gut einem Drittel der Betriebe beobachtet jedoch eine gestiegene Bereitschaft zu Zugeständnissen. Es ist erkennbar, dass die gestiegene Konzessionsbereitschaft im Jahr 2005 im Vergleich zu 2004 als insgesamt höher eingeschätzt wird als im Folgejahr 2006 gegenüber 2005. Die Einführung der Reform könnte das Verhalten also beeinflusst haben.

\section{Spezifikation der Regres- sionsmodelle}

Um die seitens der Betriebe wahrgenommene Konzessionsbereitschaft zu untersuchen, wurden vier logistische Regressionsmodelle geschätzt. Die ersten drei analysieren die Chance, mit der Betriebe ein geändertes Verhalten arbeitsloser Bewerber beobachten. Berücksichtigt wurden die Antworten aller Betriebe, die in den vorangegangenen zwölf Monaten Neueinstellungen vorgenommen haben.

Das vierte Modell untersucht die Chance, dass Betriebe das Verhalten ihrer Belegschaft als geändert einschätzen. In diesem Modell werden alle Betriebe berücksichtigt. Insgesamt stehen die Antworten von jeweils knapp 7.000 Betrieben zur Verfügung.

\subsection{DIE ABHÄNGIGE VARIABLE: GESTIEGENE KONZESSIONS- BEREITSCHAFT}

Um die beobachteten Verhaltensänderungen arbeitsloser Bewerber zu untersuchen, wurden die drei Variablen zur Bereitschaft, einen Arbeitsplatz unterhalb der eigentlichen Qualifikation anzunehmen, Zugeständnisse bei der Entlohnung zu machen und schwierigere Arbeitsbedingungen zu akzeptieren, zu Dummyvariablen umkodiert. Die Variablen nehmen den Wert 1 an, wenn die Betriebe angaben, dass die Bereitschaft, Konzessionen einzugehen, gestiegen ist. Sie nehmen den Wert 0 an, wenn die Bereitschaft als unverändert oder gesunken bewertet wurde. Es entstehen somit drei Variablen für je eine Antwortmöglichkeit auf die Frage nach Verhaltensänderungen arbeitsloser Bewerber.

Bei den Beschäftigten ist Konzessionsbereitschaft schwerer zu operationalisieren. Da es sich hier um ein bestehendes Arbeitsverhältnis handelt, kann von der Zustimmung zu einem Item der Fragenbatterie nicht automatisch auf Konzessionsbereitschaft geschlossen werden. So tritt erhöhte Konzessionsbereitschaft beispielsweise dann auf, wenn der Betrieb eine gestiegene Bereitschaft beobachtete, Einschränkungen bei der Entlohnung zu akzeptieren und gleichzeitig die Bereitschaft zu höherer Leistung oder geänderten Arbeitszeiten als unverändert oder gestiegen 
wahrgenommen wird. Beobachtet ein Betrieb zwar eine gestiegene Bereitschaft, geringere Löhne zu akzeptieren, gleichzeitig aber eine gesunkene Bereitschaft zu höherer Leistung oder geänderten Arbeitszeiten, so wird dies nicht als Anstieg der Konzessionsbereitschaft bewertet. Aufgrund dessen war für die Untersuchung der Konzessionsbereitschaft Beschäftigter ein anderes Vorgehen nötig. Anhand der drei möglichen Antwortkategorien wurde eine Variable entwickelt, die für eine gestiegene Konzessionsbereitschaft den Wert 1, andernfalls den Wert 0, annimmt. Es wird definiert, dass Betriebe dann eine gestiegene Konzessionsbereitschaft der Beschäftigten beobachten, wenn wenigstens eine der drei Fragen zu Leistungsbereitschaft, Arbeitszeit und Einschränkung bei der Entlohnung mit „gestiegen“ beantwortet wurde und gleichzeitig keine der anderen mit „gesunken“.

\subsection{SPEZIFIKATION DER ERKLÄREN- DEN VARIABLEN}

\section{MAKROÖKONOMISCHE VARIABLEN}

Mit der Arbeitsmarktanspannung in einer Region wird das Verhältnis offener Stellen zu Arbeitslosen gemessen (Pissarides 2000). Nimmt dieses Verhältnis einen hohen Wert an, stehen viele offene Stellen einer vergleichsweise geringen Zahl Arbeitsloser gegenüber. Diese Situation ist für Arbeitsuchende günstig, und es ist zu erwarten, dass die Konzessionsbereitschaft arbeitsloser Bewerber dann eher nicht steigt. Ein weiterer Indikator für die Arbeitsmarktlage ist der Anteil der Arbeitslosen im Rechtskreis SGB II an allen Arbeitslosen, da der größte Teil dieser Personen langzeitarbeitslos ist. In einer Region, die einen hohen Anteil Langzeitarbeitsloser aufweist, stehen die Arbeitslosen ebenso wie die Beschäftigten unter einem größeren Druck (vgl. Clark et al. 2009), da der Arbeitsmarkt weniger Möglichkeiten bietet.

In das Modell wurden die Veränderungen der Reallöhne zwischen 2004 und 2005 sowie zwischen 2005 und 2006 aufgenommen. Es ist zu erwarten, dass die Entwicklung der Reallöhne in den einzelnen Regionen Einfluss auf Verhaltensänderungen hat. Eine zunehmende Bereitschaft, Einschränkungen zu akzeptieren, sollte in solchen Regionen geringer sein, in denen die Reallöhne niedrig sind, da hier die Spannweite, innerhalb derer Konzes- sionen möglich sind, geringer ausfallen müsste.

Die Veränderungsrate des Bruttoinlandsprodukts (BIP) repräsentiert die ökonomische Entwicklung. Eine negative oder nur leicht positive Veränderungsrate des BIP geht in der Regel mit einer geringeren Arbeitskräftenachfrage einher und erschwert damit die Arbeitsplatzsuche. Es ist zu erwarten, dass Firmen in solchen Regionen eher eine gestiegene Konzessionsbereitschaft arbeitsloser Bewerber und Beschäftigter beobachten.

\section{BETRIEBSSPEZIFISCHE VARIABLEN}

Ferner ist anzunehmen, dass die Einstellungsquote eines Betriebes und die Quote der betriebsbedingten Kündigungen Einfluss auf die betriebliche Einschätzung der Konzessionsbereitschaft beider Personengruppen haben. Eine hohe Neueinstellungsrate und eine geringe Rate betriebsbedingter Kündigungen sprechen für eine stabile ökonomische Situation des Betriebes. In diesem Fall ist eine höhere Bereitschaft zu Zugeständnissen einerseits unwahrscheinlicher. Andererseits kann sich bei einer hohen Neueinstellungsrate die Konkurrenz zwischen den Bewerbern zum einen und die zwischen Neueinsteigern und Beschäftigten zum anderen verschärfen. Eine gestiegene Konzessionsbereitschaft in den Gruppen wäre dann zu erwarten.

Des Weiteren ist zu vermuten, dass Betriebe, die einen hohen Anteil an gering qualifiziertem Personal beschäftigen, eher eine gestiegene Konzessionsbereitschaft beobachten. Die Personalfluktuation (Labour-Turnover-Rate) ist bei dieser Personengruppe in Deutschland etwa doppelt so hoch wie bei Personen mit abgeschlossener Berufsausbildung (Bachmann/David 2009). Ein Grund dafür mag in der Charakteristik der Stellen liegen. Personen mit geringer Qualifikation werden eher für Arbeitsplätze mit geringem Einarbeitungsaufwand eingestellt und sind somit schneller „austauschbar“, was die Arbeitsplatzunsicherheit dieser Beschäftigten erhöhen dürfte. Darüber hinaus ist das Risiko, langzeitarbeitslos zu werden, hier besonders hoch (Reinberg/Hummel 2007; Gartner/Klinger 2008), was nach Jahresfrist zu geringen Anspruchslöhnen führt.

Auch der Anteil befristet Beschäftigter im Betrieb kann zur Wahrnehmung gestiegener Konzessionsbereitschaft führen. Das resultiert aus der höheren Ar- beitsplatzunsicherheit dieser Personen gegenüber Beschäftigten mit unbefristeten Arbeitsverträgen (De Witte/Näsvall 2003). Auch ist bekannt, dass sie häufig überdurchschnittliche Leistung wie unbezahlte Überstunden erbringen (Engellandt/Riphahn 2005).

Zwei weitere Variablen können Hinweise auf die ökonomische Situation eines Betriebes geben: Es wurde eine Dummyvariable integriert, die den Wert 1 annimmt, wenn der Betrieb in den vorangegangenen zwölf Monaten finanzielle Schwierigkeiten hatte. Betriebe die darüber berichten, sollten eher Erfahrungen mit konzessionsbereiten Beschäftigten machen. Zum anderen wurde eine Dummyvariable aufgenommen, die den Wert 1 annimmt, wenn der Betrieb in den kommenden zwölf Monaten mit einem Beschäftigtenzuwachs rechnet. Für diese Variable wird der gegenteilige Effekt erwartet, da solche Betriebe weniger auf Zugeständnisse angewiesen sind.

Der Stichprobenstruktur folgend werden sechs Dummyvariablen für die Wirtschaftszweigklassifikation 2003, vier Dummyvariablen für die Betriebsgrößenklassen und ein Dummy für Westdeutschland integriert.

Nachdem für die wirtschaftlichen Einflüsse in den Bundesländern kontrolliert wurde, soll eine Dummyvariable für das Jahr 2005 Hinweise auf mögliche Reformeffekte geben. Die konkreten Auswirkungen der Hartz-IV-Reform auf das Verhalten von Arbeitslosen und Beschäftigten können hier nicht exakt zugeordnet werden. Als Reformwirkung erwarten wir jedoch einen Anstieg der beobachteten Konzessionsbereitschaft.

\section{Ergebnisse}

\subsection{DAS VERHALTEN ARBEITSLOSER BEWERBER}

Inwiefern Betriebe eine gestiegene Konzessionsbereitschaft arbeitsloser Bewerber in den Jahren 2005 und 2006 beobachtet haben, ist in Tabelle 3 dargestellt. Im ersten Modell werden die Chancen abgebildet, dass ein Betrieb eine gestiegene Bereitschaft zu unterwertiger Arbeit beobachtet. Das zweite Modell gibt die betrieblichen Einschätzungen einer gestiegenen Bereitschaft zu Zugeständnissen bei der Entloh- 
Tabelle 3: Betriebliche Einschätzungen zur veränderten Konzessionsbereitschaft arbeitsloser Bewerber Logistische Regressionen

\begin{tabular}{|c|c|c|c|c|c|c|c|c|c|}
\hline \multirow[t]{2}{*}{ Arbeitslose Bewerber zeigen eine gestiegene Bereitschaft } & \multicolumn{3}{|c|}{$\begin{array}{l}\text {...Arbeitsplätze unterhalb ihrer } \\
\text { Qualifikation anzunehmen }\end{array}$} & \multicolumn{3}{|c|}{$\begin{array}{l}\text {...Zugeständnisse bei der } \\
\text { Entlohnung zu machen }\end{array}$} & \multicolumn{3}{|c|}{$\begin{array}{l}\text {...schwierige Arbeits- } \\
\text { bedingungen zu akzeptieren }\end{array}$} \\
\hline & \multicolumn{2}{|c|}{ Odds Ratio } & \multirow[t]{2}{*}{ Standardfehler ${ }^{1}$} & \multicolumn{2}{|c|}{ Odds Ratio } & \multirow[t]{2}{*}{ Standardfehler ${ }^{1}$} & \multicolumn{2}{|c|}{ Odds Ratio } & \multirow[t]{2}{*}{ Standardfehler ${ }^{1}$} \\
\hline Externe Einflussvariablen & & & & & & & & & \\
\hline Arbeitsmarktanspannung & 0,603 & $* * *$ & 0,096 & 0,806 & & 0,191 & 0,702 & *** & 0,077 \\
\hline Anteil Arbeitsloser im Rechtskreis SGB II & 0,774 & & 0,456 & 3,114 & & 3,092 & 1,743 & & 0,669 \\
\hline Veränderung der Reallöhne & 1,096 & ** & 0,044 & 0,994 & & 0,040 & 1,056 & & 0,038 \\
\hline Veränderung des BIP & 1,009 & & 0,034 & 1,013 & & 0,054 & 1,015 & & 0,055 \\
\hline \multicolumn{10}{|l|}{ Betriebsspezifische Einflussvariablen } \\
\hline Quote der Neueinstellungen & 1,132 & & 0,116 & 1,295 & ** & 0,132 & 1,201 & $* *$ & 0,112 \\
\hline Quote betrieblicher Entlassungen & 0,212 & $* * *$ & 0,088 & 0,477 & ** & 0,145 & 0,379 & $* * *$ & 0,097 \\
\hline Quote geringqualifizierter Beschäftigter & 1,000 & & 0,000 & 1,000 & & 0,000 & 1,000 & & 0,000 \\
\hline Quote befristet Beschäftigter & 1,062 & & 0,059 & 1,052 & & 0,049 & 1,010 & & 0,038 \\
\hline Der Betrieb hatte finanzielle Schwierigkeiten & 1,050 & & 0,050 & 1,292 & $* * *$ & 0,073 & 1,311 & *** & 0,104 \\
\hline Der Betrieb erwartet einen Beschäftigtenzuwachs & 1,142 & * & 0,090 & 1,094 & & 0,070 & 1,095 & & 0,073 \\
\hline \multicolumn{10}{|l|}{ Wirtschaftszweige } \\
\hline \multicolumn{10}{|l|}{ Referenz: Verarbeitendes Gewerbe } \\
\hline Landwirtschaft und Fischerei & 0,742 & * & 0,116 & 0,703 & *** & 0,086 & 0,737 & * & 0,123 \\
\hline Baugewerbe & 0,765 & * & 0,111 & 0,905 & & 0,088 & 0,961 & & 0,111 \\
\hline Handel, Gastgewerbe, Verkehr und Nachrichtenübermittlung & 0,779 & ** & 0,085 & 0,709 & $* * *$ & 0,065 & 0,874 & & 0,095 \\
\hline Banken, Versicherungen und wirtschaftliche Dienstleistungen & 0,894 & & 0,097 & 0,926 & & 0,125 & 0,848 & & 0,127 \\
\hline Private, Soziale und Öffentliche Dienstleistungen & 1,186 & * & 0,111 & 1,083 & & 0,095 & 1,156 & * & 0,090 \\
\hline \multicolumn{10}{|l|}{ Betriebsgrößenklasse } \\
\hline \multicolumn{10}{|l|}{$\begin{array}{l}\text { Referenz: Betriebe mit weniger als } 10 \text { sozialversicherungs- } \\
\text { pflichtig (SV-) Beschäftigten }\end{array}$} \\
\hline 10 bis 49 SV-Beschäftigte & 0,805 & $* * *$ & 0,053 & 0,922 & & 0,076 & 0,896 & & 0,064 \\
\hline 50 bis 199 SV-Beschäftigte & 0,840 & ** & 0,074 & 0,969 & & 0,089 & 0,930 & & 0,078 \\
\hline mehr als 200 SV-Beschäftigte & 1,019 & & 0,093 & 1,076 & & 0,085 & 0,977 & & 0,111 \\
\hline Jahr 2005 & 1,638 & $* * *$ & 0,193 & 1,559 & $* *$ & 0,283 & 1,552 & $* * *$ & 0,224 \\
\hline Westdeutschland & 1,232 & $* * *$ & 0,074 & 1,369 & $* * *$ & 0,101 & 1,363 & $* * *$ & 0,082 \\
\hline$N=$ & 5996 & & & 6016 & & & 5985 & & \\
\hline linktest hat ${ }^{2}, \mathrm{H}_{0}=$ Das Modell ist nicht korrekt spezifiziert. & 0,984 & & & 0,156 & & & 0,481 & & \\
\hline Hosmer-Lemeshow goodness of fit & 0,467 & & & 0,231 & & & 0,063 & & \\
\hline Count $R^{2}$ & 0,727 & & & 0,681 & & & 0,748 & & \\
\hline
\end{tabular}

nung wieder. Das dritte Modell zeigt die Ergebnisse hinsichtlich einer gestiegenen Bereitschaft, schwierige Arbeitsbedingungen zu akzeptieren.

Der Anstieg der Arbeitsmarktanspannung verringert die Chance, dass ein Betrieb eine gestiegene Bereitschaft arbeitsloser Bewerber beobachtet, eine unterwertige Beschäftigung anzunehmen oder schwierige Arbeitsbedingungen zu akzeptieren. Dies ist plausibel, da arbeitslose Bewerber in diesem Fall eine größere Auswahl an Stellen haben und weniger Konzessionen eingehen müssen. Steigende Reallöhne wirken hingegen schwach positiv auf die Akzeptanz unterwertiger Stellen. Die Bewerber bieten möglicherweise bei steigenden Reallöhnen ihre Arbeitskraft für eine Beschäftigung unterhalb ihrer Qualifikation an.

Die Quote der betrieblichen Neueinstellungen hat positiven Einfluss auf die Wahrnehmung von Zugeständnissen hinsichtlich geringerer Entlohnung und schwieriger Arbeitsbedingungen. Es ist denkbar, dass eine Vielzahl von Neueinstellungen die Konkurrenz unter den Bewerbern erhöht und somit zu einem gegenseitigen „Unterbieten“ führt. Die Quote betrieblicher Entlassungen wirkt negativ auf die Beobachtung von Zugeständnissen. Denkbar ist, dass diese Betriebe ein sehr hohes Maß an Zugeständnissen benötigen, um nicht entlassen zu müssen. Das würde bedeuten, dass die Konzessionsbereitschaft der Bewerber nicht als Zugeständnis wahrgenommen wird.

Betriebe, die in den vorangegangenen zwölf Monaten finanzielle Probleme hatten, beobachteten bei den arbeitslosen Bewerbern erwartungsgemäß eine gestiegene Konzessionsbereitschaft hinsichtlich der Akzeptanz geringerer Löhne und schwieriger Arbeitsbedingungen. Es ist denkbar, dass diese Zugeständnisse den arbeitslosen Bewerbern den Eintritt in den Betrieb ermöglicht haben, wobei die Option auf eine Verbesserung der Situation des Betriebes nicht ausgeschlossen ist. Erwartet ein Betrieb Beschäftigungszuwächse, so ist zu erkennen, dass er eine gestiegene Konzessionsbereitschaft hinsichtlich der Aufnahme einer unterwertigen Beschäftigung beobachtet.

Für die Jahresdummyvariable 2005 ergeben sich in allen drei Modellen positive Effekte. Dies mag ein Hinweis darauf sein, dass die Arbeitsmarktreform einen Einfluss auf Verhaltensänderungen arbeitsloser Bewerber hatte. Daraus folgend sehen wir Indizien dafür, dass die Reform die betriebliche Wahrnehmung von Konzessionsbereitschaft beeinflusst hat.

\subsection{DAS VERHALTEN BESCHÄFTIGTER}

In Tabelle 4 sind die Ergebnisse der betrieblichen Einschätzung gestiegener Konzessionsbereitschaft der Beschäftigten dargestellt. Ähnlich wie in den vorangegange- 


\begin{tabular}{|c|c|c|c|}
\hline \multirow{2}{*}{$\begin{array}{l}\text { Tabelle 4: Betriebliche Einschätzungen zur } \\
\text { bereitschaft von Beschäftigten - Logistisch } \\
\text { Beschäftigte zeigen eine gestiegene Konzessionsbereitschaft }{ }^{1} \\
\text { Externe Einflussvariablen }{ }^{3}\end{array}$} & \multicolumn{2}{|c|}{ Odds Ratio } & \multirow[t]{2}{*}{ Standardfehler ${ }^{2}$} \\
\hline & & & \\
\hline Arbeitsmarktanspannung & 0,626 & $* * *$ & 0,109 \\
\hline Anteil Arbeitsloser im Rechtskreis SGB II & 0,965 & & 0,479 \\
\hline Veränderung der Reallöhne & 1,064 & * & 0,036 \\
\hline Veränderung des BIP & 1,013 & & 0,046 \\
\hline \multicolumn{4}{|l|}{ Betriebsspezifische Einflussvariablen } \\
\hline Quote der Neueinstellungen & 0,943 & & 0,085 \\
\hline Quote betrieblicher Entlassungen & 0,851 & & 0,148 \\
\hline Quote geringqualifizierter Beschäftigter & 1,000 & & 0,000 \\
\hline Quote befristet Beschäftigter & 1,079 & & 0,061 \\
\hline Der Betrieb hatte finanzielle Schwierigkeiten & 1,335 & $* * *$ & 0,057 \\
\hline Der Betrieb erwartet einen Beschäftigtenzuwachs & 1,241 & $* * *$ & 0,065 \\
\hline \multicolumn{4}{|l|}{ Wirtschaftszweige } \\
\hline \multicolumn{4}{|l|}{ Referenz: Verarbeitendes Gewerbe } \\
\hline Landwirtschaft und Fischerei & 0,868 & * & 0,074 \\
\hline Baugewerbe & 0,840 & & 0,095 \\
\hline Handel, Gastgewerbe, Verkehr und Nachrichtenübermittlung & 0,889 & & 0,075 \\
\hline Banken, Versicherungen und wirtschaftliche Dienstleistungen & 0,840 & * & 0,088 \\
\hline Private, Soziale und Öffentliche Dienstleistungen & 0,790 & ** & 0,079 \\
\hline \multicolumn{4}{|l|}{ Betriebsgrößenklasse } \\
\hline \multicolumn{4}{|l|}{$\begin{array}{l}\text { Referenz: Betriebe mit weniger als } 10 \text { sozialversicherungspflichtig } \\
\text { (SV-) Beschäftigten }\end{array}$} \\
\hline 10 bis 49 SV-Beschäftigte & 0,785 & $* * *$ & 0,061 \\
\hline 50 bis 199 SV-Beschäftigte & 0,856 & $* * *$ & 0,036 \\
\hline mehr als 200 SV-Beschäftigte & 1,007 & & 0,086 \\
\hline Jahr 2005 & 1,331 & $* *$ & 0,193 \\
\hline Westdeutschland & 1,490 & $* * *$ & 0,093 \\
\hline$N=$ & 6893 & & \\
\hline linktest hat ${ }^{2}, \mathrm{H}_{0}=$ Das Modell ist nicht korrekt spezifiziert. & 0,192 & & \\
\hline Hosmer-Lemeshow goodness of fit & 0,018 & & \\
\hline Count $\mathrm{R}^{2}$ & 0,591 & & \\
\hline \multicolumn{4}{|c|}{$\begin{array}{l}1 \text { Die einzelnen Elemente der Konzessionsbereitschaft (Bereitschaft zu höherer Leistung, zu längeren oder geänderten Ar- } \\
\text { beitszeiten und zu Einschränkungen bei der Entlohnung) wurden anhand einzelner logistischer Regressionen getestet. Dabei } \\
\text { spielen makroökonomische Variablen kaum und der Jahresdummy keine Rolle. Es wurden verschiedene Varianten des hier } \\
\text { besprochenen Modells geschätzt, ohne dass Veränderungen in der Aussage auftraten. Die Schätzergebnisse werden von den } \\
\text { Autorinnen gern zur Verfügung gestellt. } \\
2 \text { Um für die Intragruppenkorrelationen der Bundesländer zu korrigieren, wurden robuste Standardfehler berechnet. } \\
{ }^{3} \text { Alle makroökonomischen Variablen wurden auf Bundeslandebene integriert. } \\
\text { * signifikant mit } 10 \% ; * * \text { signifikant mit } 5 \% \text {; *** signifikant mit } 1 \% \text {. }\end{array}$} \\
\hline Quelle: Berechnungen der Autorinnen. & & & MITTEILUNGEN \\
\hline
\end{tabular}

nen Modellen zeigt auch hier die Arbeitsmarktanspannung einen negativen Einfluss und steigende Reallöhne zeigen einen positiven. Sind verhältnismäßig viele offene Stellen vorhanden, so müssen auch die Beschäftigten nicht konzessionsbereit sein, da sie die Möglichkeit haben, in einen anderen Job zu wechseln. Bei steigenden Reallöhnen ist die Konzessionsbereitschaft der Belegschaft möglicherweise deshalb wahrscheinlicher, weil der Verlust des Arbeitsplatzes höhere Lohneinbußen mit sich bringen würde.

Im Hinblick auf betriebsspezifische Einflussvariablen lassen sich nur zwei Effekte identifizieren: Lediglich dann, wenn ein Betrieb finanzielle Schwierigkeiten hatte oder einen Beschäftigungszuwachs erwartet, beobachten die Betriebe mit einer höheren Wahrscheinlichkeit eine Bereitschaft zu Zugeständnissen. Beide Ergebnisse sind plausibel. In einer betrieblich schwierigen Phase werden Beschäftigte eher Zugeständnisse machen, um ihren Arbeitsplatz zu sichern. Baut der Betrieb Beschäftigung auf, so ist denkbar, dass sich eine Situation entwickelt, in der Beschäftigte in Konkurrenz zueinander treten oder dass ihre Zugeständnisse es dem Betrieb erst ermöglichen, Beschäftigung aufzubauen.

Für das Jahr 2005 konnte ebenfalls ein positiver Effekt identifiziert werden. Es ist also denkbar, dass die Neuregelungen im Rahmen der Hartz-Reformen verstärkend auf ein arbeitsplatzsicherndes Verhalten von Beschäftigten gewirkt haben könnten.

\section{Fazit}

Ziel dieses Aufsatzes war es, Determinanten einer von Betrieben beobachteten gestiegenen Konzessionsbereitschaft arbeitsloser Bewerber und Beschäftigter nach den HartzReformen zu identifizieren. Um zweifelsfreie Resultate zu den direkten Auswirkungen der Arbeitsmarktreformen zu erhalten, bedarf es Analysen, die sich auf längere Zeitreihen stützen. Obwohl es nur über diesen Weg möglich ist, Konjunktureffekte von Reformeffekten zu differenzieren, kann dieser Beitrag dennoch Indizien aufzeigen.

Mit Daten der Erhebung des gesamtwirtschaftlichen Stellenangebots wurde untersucht, inwiefern Betriebe das Verhalten arbeitsloser Bewerber und Beschäftigter als verändert gegenüber dem Vorjahr im Sinne einer erhöhten Konzessionsbereitschaft wahrgenommen haben.

Sowohl bei arbeitslosen Bewerbern als auch bei Beschäftigten waren makroökonomische und betriebsinterne Faktoren für die betriebliche Einschätzung einer gestiegenen Konzessionsbereitschaft verantwortlich. Ist die Situation am Arbeitsmarkt gut, werden beide Gruppen weniger konzessionsbereit sein. Für arbeitslose Bewerber ist es dann nicht nötig, den „erstbesten“ Arbeitsplatz anzunehmen und möglicherweise hinter ihren Reservationslohn zurückzufallen. Gleichzeitig können steigende Reallöhne und höherer Konkurrenzdruck die Konzessionsbereitschaft insofern erhöhen, als dass qualitative Abstriche bei der Jobsuche gemacht werden. Mit Blick auf die bereits Beschäftigten ist gestiegene Konzessionsbereitschaft vor allem dann wahrscheinlicher, wenn die Arbeitsplatzunsicherheit aufgrund betrieblicher Schwierigkeiten zunimmt oder sich der Konkurrenzdruck erhöht. Sollten die Hartz-Reformen zu einer höheren Suchintensität geführt haben, so wären die Hinweise auf gestiegene Konzessionsbereitschaft aufgrund von Konkurrenzdruck plausibel. Das wird durch die Dummyvariable für das Jahr 2005 bestärkt. Mehr als im Jahr 2006 waren Betriebe der Auffassung, eine gestiegene Bereitschaft zu Zugeständnissen zu beobachten. 
Abbring J.H./Berg, G.J./Ours, J.C. van (2005): The Effect of Unemployment Insurance Sanctions on the Transition Rate from Unemployment to Employment, in: Economic Journal, 115 (505), S. 602-630

Andersen T.M./Svarer, M. (2007): Flexicurity - Labour Market Performance in Denmark, CESifo Working Paper Series (2108), München Ashford, S.J./Lee,C./Bobko, P. (1989): Content, Causes, and Consequences of Job Insecurity: A Theory-Based Measure and Substantive Test, in: The Academy of Management Journal 32 (4), S. 803-829 Bachmann, R./David P. (2009): The Importance of Two-Sided Heterogeneity for the Cyclicality of Labour Market Dynamics, Ruhr Economic Papers (0124) hrsg. von: Rheinisch-Westfälisches Institut für Wirtschaftsforschung/Ruhr-Universität Bochum/Universität Dortmund/ Universität Duisburg-Essen

Bender, S./Koch, S./Meßmann, S./Walwei, U. (2008): Was muten sich Arbeitslose zu? Lohnkonzessionen von ALG-II-Empfängern,

in: Sozialer Fortschritt 57 (3), S. 75-85

Bosch, G./Kalina, T./Weinkopf, C. (2008): Niedriglohnbeschäftigte auf der Verliererseite, in: WSI-Mitteilungen 61 (8), S. 423-430

Braun, T. (2001): Arbeitsmarktpolitik in Dänemark, FES-Analyse, hrsg. von der Friedrich-Ebert-Stiftung, Bonn

Christensen, B. (2003):Anspruchslohn und Arbeitslosigkeit in Deutschland, Mitteilungen aus der Arbeitsmarkt- und Berufsforschung (4),

S. 573-598

Clark, A.E./Knabe, A./Rätzel, S. (2009) Boon or Bane? Others' Unemployment, Well-being and Job Insecurity, IZA Discussion Papers (4210), Bonn

Decressin, A. (2001): Tinkering with or Tackling Unemployment Compensation?, Georgetown University Working Paper (01-05), Washington D.C.

De Witte, H./Näswall, K. (2003): 'Objective' vs 'Subjective' Job Insecurity: Consequences of Temporary Work for Job Satisfaction and Organizational Commitment in Four European Countries, in: Economic and Industrial Democracy 24 (2), S. 149-188

Engellandt, A./Riphahn, R.T. (2005): Temporary contracts and employee effort, in: Labour Economics 12 (3), S. 281-299

Fahr, R./Sunde, U. (2009): "Did the Hartz Reforms Speed-Up the Matching Process? A Macro-Evaluation Using Empirical Matching Functions“", in: German Economic Review 10 (3), S. 284-316

Fitzenberger, B./Wilke, R.A. (2009): Unemployment Durations in West Germany before and after the Reform of the Unemployment Compensation System during the 1980s, in: German Economic Review 11 (3), S. 336-366

Gartner, H./Klinger, S. (2008): Ein janusköpfiger Aufschwung. Beschäftigungsgewinne und Polarisierung, in: WSI-Mitteilungen 61 (8), S. $439-446$
Geerdsen, L.P. (2006): Is there a Threat Effect of Labour Market Programmes? A Study of ALMP in the Danish UI System, in: The Economic Journal 116 (513), S. 738-750

Greenhalgh, L./Rosenblatt, Z. (1984): Job Insecurity: Toward Conceptual Clarity, in: The Acadamy of Management Review 9 (3), S. $438-448$

Hunt, J. (1995): The Effect of the Unemployment Compensation on Unemployment Duration in Germany, in: Journal of Labor Economics 13 (1), S. 88-120

International Labour Organization (ILO) (2008): Global Wage Report 2008/09: Minimum wages and collective bargaining: Towards policy coherence, Genf

Jacobi, L./Kluve, J. (2007): Before and after the Hartz Reforms: The performance of active labour market policy in Germany, in: Journal of Labour Market Research 40 (1), S. 45-64 Kalina, T./Weinkopf, C. (2008): Weitere Zunahme der Niedriglohnbeschäftigung: 2006 bereits rund 6,5 Millionen Beschäftigte betroffen, IAQ-Report (1), Essen

Kettner, A./Heckmann, M./Rebien, M./Pausch, S./Szameitat, J. (2011): Die IAB-Erhebung des gesamtwirtschaftlichen Stellenangebots Inhalte, Daten und Methoden, in: Zeitschrift für Arbeitsmarktforschung (im Erscheinen)

Kettner A./Rebien, M. (2007): Hartz IV-Reform: Impulse für den Arbeitsmarkt, IAB-Kurzbericht (19), Nürnberg

Kettner, A./Spitznagel, E. (2006): Gesamtwirtschaftliches Stellenangebot: Kräftiger Anstieg nach jahrelangem Rückgang, IAB-Kurzbericht (06), Nürnberg

Klinger, S./Rothe, T. (2010): The impact of labour market reforms and economic performance on the matching of short-term and long-term unemployed, IAB Discussion Paper (13), Nürnberg

Lindbeck, A./Snower, D.J. (2001): Insiders versus Outsiders, in: The Journal of Economic Perspectives 15 (1), S. 165-188 Pissarides, C. (2000): Equilibrium unemployment theory, Cambridge/London

Reinberg, A./Hummel, M. (2007): Schwierige Fortschreibung: Der Trend bleibt. Geringqualifizierte sind häufiger arbeitslos, IAB-Kurzbericht (18), Nürnberg

Schramm, F. (1992): Beschäftigungsunsicherheit. Wie sich die Risiken des Arbeitsmarktes auf die Beschäftigung auswirken - Empirische Analysen in Ost und Westdeutschland, Beiträge zur Sozialökonomik der Arbeit Bd. 25, München Svarer, M. (2007): The Effect of Sanctions on the Job Finding Rate: Evidence from Denmark, IZA Discussion Papers (3015), Bonn 